\title{
INFLUENCE D'UN CHAMP MAGNÉTIQUE SUR LE RAYONNEMENT DES CORPS RADIO-AGTIFS ;
}

Par M. Henri BeGQuerei.

Au cours des expériences que je poursuis dans la recherche des faits qui peuvent nous éclairer sur la nature du rayonnement émis par les corps radio-actifs, j'ai eu l'occasion d'observer récemment des effets remarquables produits sur ce rayonnement lorsqu'il traverse un champ magnétique.

Dans un champ magnétique non uniforme, constitué par un puissant électro-aimant, j’ai observé que le rayonnement du radium s'infléchit et se concentre sur les pôles.

M. et $\mathrm{M}^{\mathrm{me}}$ Curie, à qui je montrais quelques-unes des expériences qui vont être décrites, m'ont alors appris que MM. Stefan Meyer et Egon R. v. Schweidler venaient de publier, trois semaines(1) auparavant, l'observation d'une action d'un champ magnétique sur les rayons du radium et du polonium, et ils m'ont communiqué la Note de ces auteurs. Ceux-ci, en étudiant dans un champ magnétique uniforme le rayonnement du radium au moyen d'un écran de platinocyanure de baryum, ont conclu de leurs expériences que, dans un plan perpendiculaire au champ, les rayons sont infléchis vraisemblablement suivant des lignes circulaires, tandis qu'il existerait dans la direction du champ des rayons qui ne sont pas déviés. Ces propriétés sont celles des rayons cathodiques. Le même phénomène avait été vu peu de temps avant par M. Giesel.

Parmi les faits que j'ai observés, sans avoir eu connaissance de ces expériences, les uns sont conformes à ceux qui ont été découverts par les auteurs allemands; les autres apportent une contribution nouvelle à nos connaissances sur le rayonnement du radium.

Voici les expériences que j'ai réalisées avec la petite quantité de chlorure de baryum radio-actif, que je dois à l'obligeance de $\mathrm{M}$. et. de $\mathrm{M}^{\mathrm{me}}$ Curie :

Effets produits dans le sens des lignes de force. - Dans un champ magnétique formé par deux petites surfaces de fer doux, planes et circulaires, de 14 millimètres environ de diamètre, qu'un fort électro-aimant permettait d’aimanter presque à saturation, et dont la

(1) Physikalische Zeitschrift. $\mathrm{n}^{\circ} 10$, p. 113-114. 
distance a varié de quelques millimètres à plusieurs centimètres, suivant les expériences, j'avais disposé d'abord, près du centre d'une des surfaces polaires, la matière radio-active, déposée sur du papier et recouverte d'une mince feuille d'aluminium battu, puis contre l'autre pôle un écran fluorescent, soit de sulfate double d'uranium et de potassium, soit de platinocyanure de baryum, de blende hexagonale, de diamant, ou d'autres corps phosphorescents.

Tant que l'électro-aimant n'est pas excité, la phosphorescence apparaît sous la forme d'une large tache lumineuse s'étendant bien audelà de la surface polaire en contact avec l'écran; la lueur est assez faible lorsque les armatures sont à une distance de $\mathbf{1}$ centimètre environ, conformément aux expériences que j'ai publiées récemment.

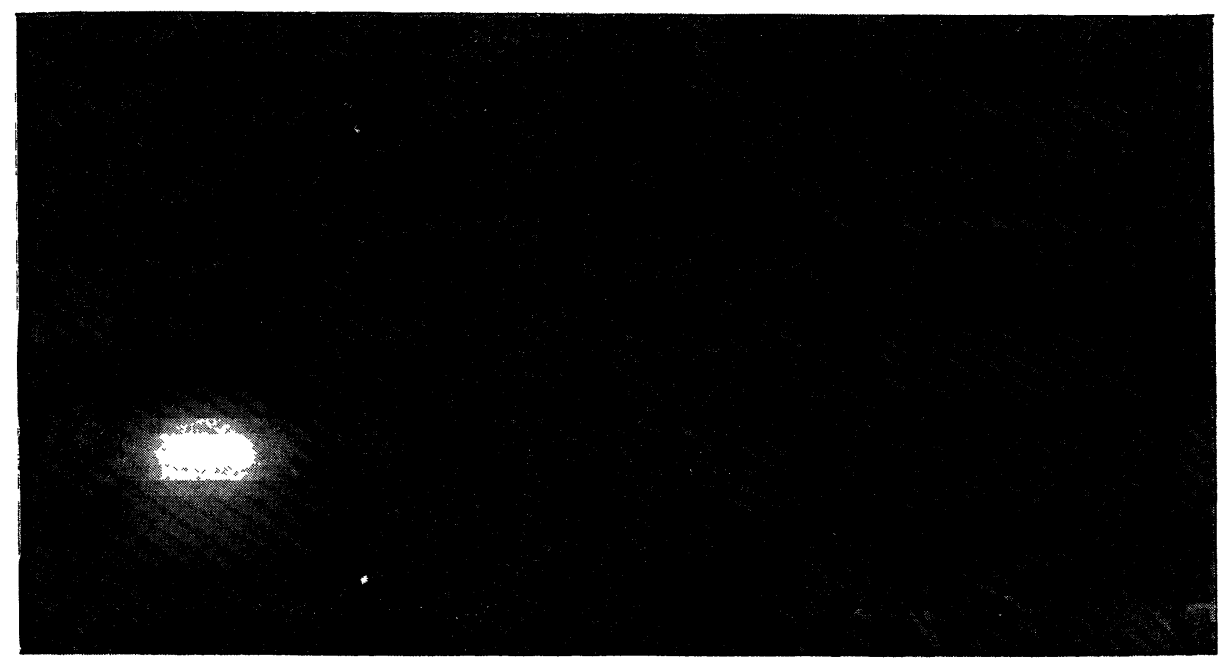

Fig. 1.

Si, alors, on excite l'électro-aimant, on voit la tache lumineuse se rétrécir et devenir considérablement plus intense. La lueur dépasse à peine les contours de la pièce polaire, et il semble que tous les rayons excitateurs émanés du radium viennent aboutir sur cette surface. L'augmentation d'intensité est considérable; elle ne présente pas de différence notable lorsqu'on change le sens de l'aimantation.

Le même phénomène se produit lorsqu'on interpose entre la matière active et la surface fluorescente des écrans de diverses 
natures, tels que du papier noir, du verre, etc. ; l'intensité s'est seulement affaiblie, comme je l'ai indiqué antérieurement.

Si aux écrans phosphorescents on substitue contre la surface polaire une plaque photographique, on obtient en quelques instants des épreuves très intéressantes. L'épreuve reproduite ci-contre ( $f g .1$ ), a été obtenue à une distance de 10 millimètres avec une minute et demie de pose. On voit sur la même plaque l'impression très faible lorsque l'électro-aimant n'est pas excité, et l'impression très intense produite par les rayons qui ont traversé le champ magnétique.

L'augmentation considérable de l'intensité, obtenue dans celte expérience donne un moyen de concentrer sur une petite surlace polaire l'action produite par une large surface de matière peu active l'expérience réussit avec des sels de radium faiblement actifs, mais je n'ai pu jusqu'ici obtenir aucun effet avec l'uranium soit en raison de la faiblesse de l'intensité, soit en raison des propriétés particulières des rayons de l'uranium.

Effets produils dans un plan perpendiculaire au champ. - Ia matière active recouverte d'aluminium, comme dans les expériences précédentes, était placée au nilieu du champ; autour de celle-ci on avait disposé un écran fluorescent cylindrique en enduisant de sulfate double d'uranium et de potassium l'intérieur d'un tube de verre dont l'axe, parallèle au champ, passait par la source radiante; tous les points de l'écran étaient ainsi sensiblement à la même distance de la source et dans une direction normale au champ. Si l'on excite alors l'électro-aimant, on voit, dans les conditions particulières de l'expérience, la phosphorescence diminuer considérablement, au point de devenir à peine perceptible.

Dans une autre expérience, la matière a été placée en dessous de la partie la plus intense du champ, et un écran fluorescent plan a été disposé au dessus, de l'autre côté du champ. Dans ces conditions, on voit l'effet changer avec le sens du courant; pour un sens de l'aimantation, la phosphorescence s'affaiblit; pour le sens contraire, elle se présente en une tache lumineuse plus brillante.

Exploration du champ par la photographie. - La photographie, par la netteté des détails qu'elle enregistre, a donné pour l'étude du champ des résultats bien supérieurs à ceux de la fluorescence. J'ai réalisé en particulier les expériences suivantes :

$1^{\circ}$ On place parallèlement au champ, entre les deux pôles distants 
de 45 millimètres, une plaque photographique horizontale entourée de papier noir. Après avoir excité l'électro-aimant, on met sur la plaque, à égale distance des pôles, la matière radio-active. Après quelques minutes de pose, on peut développer la plaque et l'on constate que l'impression, très forte, au lieu de s'être faite uniformément autour de la source, est tout entière rejetée sur la droite du champ (à gauche pour un observateur qui regarde le pôle + ). En dehors de la tache qui marque la place de la source radiante, l'impression maximum est répartie sur une zone étroite qui affecte la forme ci-contre (ffg. 2). L'écart maximum correspond à la

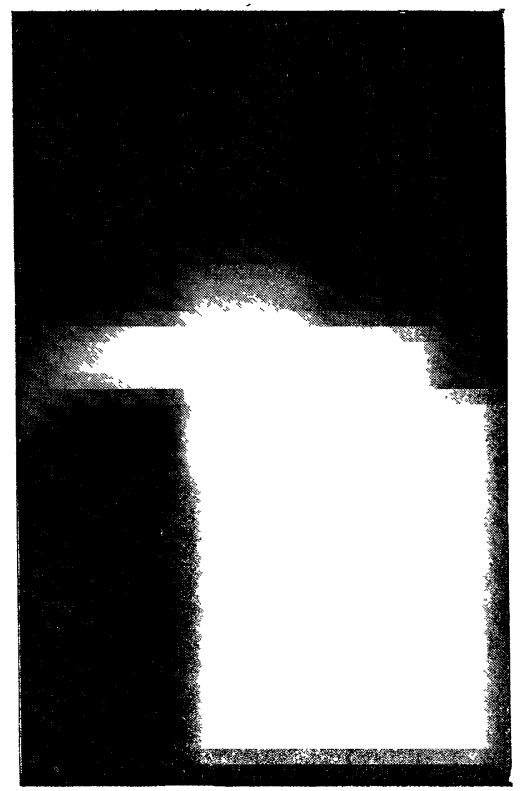

Fifr. 2.

direction normale au champ, passant par la source qui, dans le cas actuel, est au milieu du champ. Le maximum d'écart est aussi, dans ce cas, le maximum de l'intensité de l'impression ; de part et d'autre de ce maximum la courbe s infléchit et rejoint les pôles presque normalement aux surfaces polaires, en des points situés à droite des centres de ces surfaces.

La courbure est d'autant moins accentuée que le champ est plus intense. 
Cette expérience est identique à celle que MM. Meyer et Schweidler ont faite avec un écran fluorescent. Ces auteurs ont signalé seulement le maximum du sommet de la courbe; mais ils ont montré ce fait capital que l'impression était due à des rayons émis au-dessus de la plaque et ramenés sur celle-ci par l'aimant.

Les rayons émis au-dessous de la plaque donneraient la courbe symétrique; mais dans notre expérience, ils sont absorbés par le verre qu'ils ont à traverser deux fois.

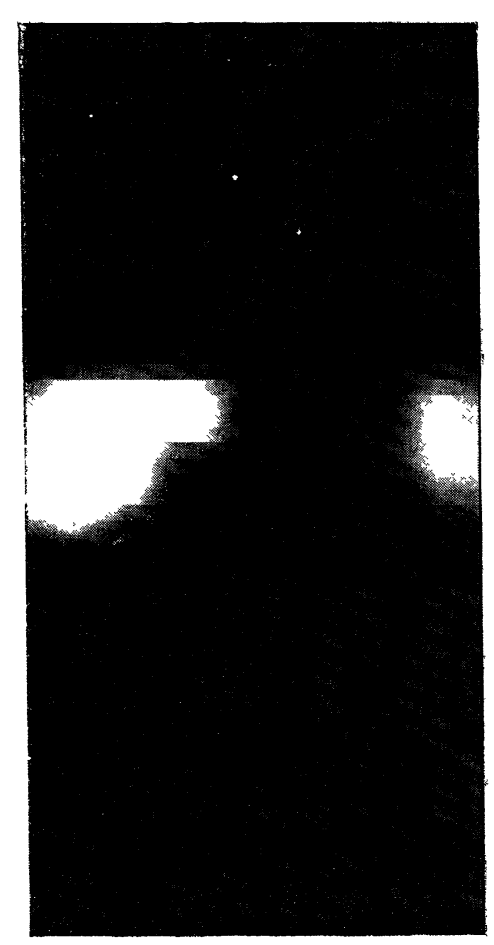

Fig. 3.

$2^{\circ}$ Une seconde expérience, tout aussi importante, consiste à placer le radium près de l'un des pôles, la plaque photographique restant dans la même position que ci-dessus. L'effet obtenu est notablement différent. L'impression affecte encore la forme curviligne décrite plus haut et est tout entière à droite du champ ( $f g .3)$. A côté de la tache marquant la place de la source, se trouve, à droite du champ, une tache presque aussi intense qui se rejoint à la première; l'im- 
pression va ensuite en diminuant le long de la courbe jusqu'au sommet, au milieu du champ, où elle est minimum, puis elle augmente en se rapprochant de l'autre pôle, près duquel elle est très intense, moins cependant qu'à l'autre extrémité, figurant une sorte d'image dispersée de la matière active.

Ces expériences montrent d'une autre manière l'augmentation d'action près des pôles, que j'ai indiquée au début de cette Note. $3^{\circ}$ Si l'on fait une troisième expérience en disposant la plaque photographique comme ci-dessus et en plaçantla matière active non plus au milieu du champ ou près d'un des pôles, mais en un point quelconque de l'axe du champ, on obtient encore la courbe décrite ci-dessus; celle-ci présente alors un maximum d'intensité en face de la source radiante, sur la même normale au champ. Ce maximum a une tendance à se rapprocher du pôle le plus voisin; et, lorsque la matière active est à une petite distance de l'un des pôles, on voit apparaître un second maximum d'action près du pôle opposé,

Parmi les expériences que j’ai réalisées, il en est d'intéressantes qui montrent qu'un écran de plomb, arrêtant le rayonnement direct de la source sur une surface fluorescente, ou photographique, n'arrête pas le rayonnement curviligne dans le champ, si celui-ci peut contourner l'obstacle; le point où ce rayonnement curviligne vient frapper l'écran change alors avec le sens de l'aimantation.

Un faisceau limité par une petite ouverture et traversant un champ magnétique uniforme, normalement aux lignes de force, est dévié normalement à ces lignes. La mesure de cette déviation fera l'objet d'un travail ultérieur.

$4^{\circ}$ Dans un champ magnétique sensiblement uniforme, et dont l'intensité était voisine de 4.000 unités C. G. S., on a placé une petite quantité du sel de radium sur une plaque photographique enveloppée de papier noir, horizontale et parallèle au champ ; puis on a disposé à angle droit, normalement au champ, une seconde plaque photographique enveloppée de papier noir, et on l'a placée dans diverses expériences, à diverses distances de la source radiante et des pièces polaires de l'électro-aimant. Après quelques minutes de pose, on obtient sur la plaque horizontale les courbes qui viennent d'être décrites, puis, sur la plaque verticale, une impression intense, limitée par une courbe qui s'enroule dans le sens du courant qui produit le champ (fig. 4). L'origine de cette courbe est sensiblement au point de rencontre de la plaque verticale et d'une droite passant par la source 
et parallèle au champ, montrant que les rayons parallèles aux lignes de force ne sont pas déviés, ainsi que l'ont reconnu MM. S. Meyer et v. Schweidler. Ces rayons, rasant la.plaque horizontale, ne donnent sur celle-ci aucune impression pholographique.

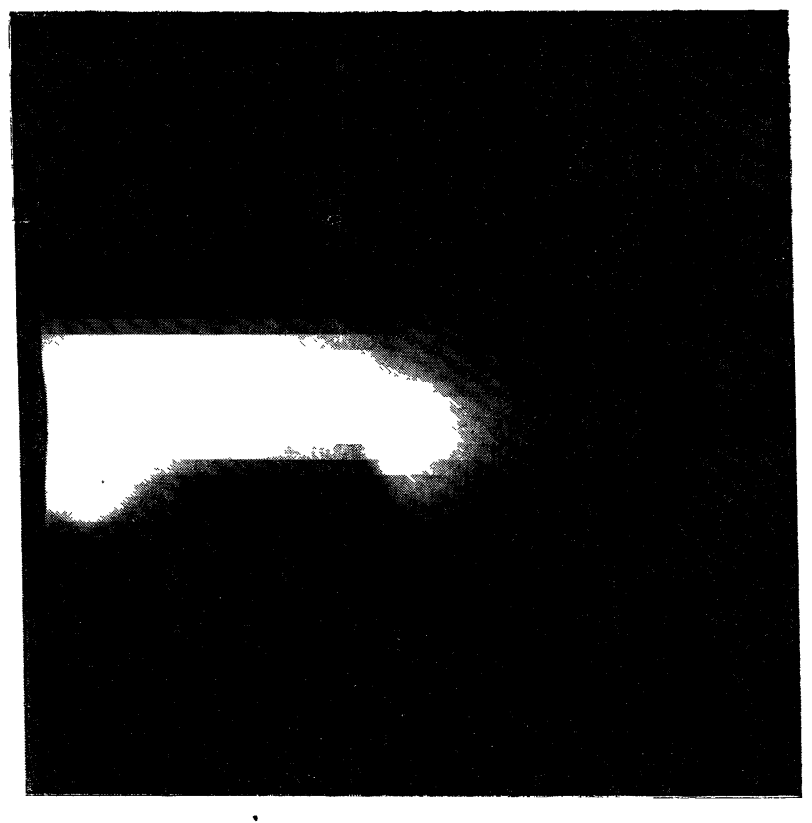

Fiti. 4.

La courbe qui limite l'impression sur la plaque verticale est l'ombre, déformée par le champ, de l'intersection des plaques horizontale et verticale, qui se comportent comme des écrans partiellement opaques. La tangente à cette courbe à l'origine fail avec l'intersection des plaques, dans le sens du mouvement d'entraînement, un angle d'autant plus grand que la distance de la source à la plaque verticale est plus grande, et que le champ est plus intense.

Une préparation de carbonate de sodium, considérablement moins active que le chlorure qui a servi aux expériences précédentes, a donné des courbes identiques à celle du chlorure dans le même champ magnétique. Le rayonnement de cette préparation ne diffère donc que par son intensité et non par sa nature.

Les expériences instituées dans le but de reconnaître si le rayon- 
nement du radium était influencé par un champ électrique ont domné des résultats négatifs.

Le rayonnement du polonium préparé par $\mathrm{M}$. el $\mathbf{M}^{\mathrm{me}}$ Curie n'a manifesté, dans les conditions des expériences précédentes, el pour des champs magnétiques atteignant 10.000 unités C. G. S., aucune déviation appréciable.

Il existe donc, dans le rayonnement des corps radio-actifs, des rayonnements de deux espèces, l'un déviable, l'autre insensible à un champ magnétique $\left({ }^{1}\right)$.

J'ai vérifié que le rayonnement non déviable du polonium donnait des ombres géométriques très nettes, comme, du reste, je l'avais reconnu dèpuis longtemps pour le rayomnenent de l'uranium, et ultérieurement pour celui du radium.

L'ensemble de ces faits, qui viennent s'ajouter à ceux que j'avais observés avec l'uranium, ne nous fixe pas encore sur la nature de ce rayonnement, dont la spontanéité apparente et la permanence sont toujours un mystère d'un grand intérèt.

(A suivre.) 